\title{
Methodology to Minimise the Abandonment of Web Page Navigation Using the Tabu Search Algorithm
}

\author{
Carmen Lozano \& Federico Fuentes \\ Department of Financial Economy and Accounting, Polytechnic University of Cartagena-Spain \\ Real, nº 3, Cartagena (Murcia), Spain \\ Tel: 34-968-325-611_E-mail: carmen.lozano@upct.es, federico.fuentes@upct.es
}

Received: February 16, $2011 \quad$ Accepted: March 16, $2011 \quad$ doi:10.5539/ass.v7n8p107

\begin{abstract}
This article shows an application of the metaheuristic tabu algorithm for resolution by domains to the optimisation of navigating an online banking webpage. In this algorithm a 'memory' is created that stores the last moves made in the navigation which, if repeated, produce results already explored. This 'memory' would serve to impede the pathway to those results. The properties of this algorithm make it ideal for optimising webpage navigation by reducing waiting times and 'noise' (irrelevant information) in the search for information and consequently a reduction in abandonment and a better quality of navigation is achieved.
\end{abstract}

Keywords: Tabu search algorithm, Artificial intelligence, Online banking

More and more people are making bank transactions via the Internet because of the ease, speed and security it offers for these operations. The facts that the audience, pages seen, visits and navigating time have increased for these financial pages is an unmistakable signal of the growing interest that these sites have aroused among internauts. Also increasingly, they know better the sections that interest them and how to accomplish their transactions in a direct and easy manner.

According to a study by Nielsen/Net Ratings (Note 1), 46,6\% of the Internet Spanish users utilize online banking to make financial consultations and transactions in a rapid and simple manner. About 6.4 million people visited financial websites between the months of April and June 2006, which is an increase of 49\% with respect to the same period in the previous year. Additionally during the trimester analysed users saw a total of 1,027 million financial institution webpages related directly to mortgages, accounts, pension plans, investment funds, loans etc., which was an increase of $76 \%$. Each online banking client visualized an average of 159 pages against 134 counted in the same period the year before. Every user made an average of 18.6 visits to these pages in the last period analysed, which means an increase of $17 \%$. According to the previously mentioned study, in Spain each user navigated financial and investment pages for approximately one and a half hours, which is an increase of one minute and nine seconds. However, the loss of customers is still frequent because of failures in the usability of the registration process as is shown by the high number of calls to 'call centers' and by emails that ask for help or complain about the use of the site. This effect is especially important for the clients (or users) that look through the site for the first time since at that time a bad experience can deprive the bank entities of the client's habitual operations in the future. On the other hand clients experienced in the site will make more visits to sites where tasks are carried out in a more rapid and agile manner (on sites more efficient).

Other typical problems that can appear in the process of navigation are the following: the site does not give all the information needed to make a decision, the operation is too slow and tedious; it does not allow the desired operation to be made via the Internet or the place in the site to make it can't be found; the site does not generate confidence in the results of the tasks that are to be carried out, etc. The appearance of any of these problems can provoke the interested user to abandon at any moment his navigation of the site and to return to it later, with the resulting waste of time. A system capable of registering the 'pathways' or regions explored in the previous navigation sessions, along with a deletion of the steps not needed to reach the intended end, would permit the user to improve his navigation and so avoid its abandonment.

Among the different heuristic methods and techniques to solve combinatorial problems the tabu search algorithm ('tabu search') (Glover et al., 1993) arose, in an attempt to provide local search algorithms with 'intelligence'. It 
is a metaheuristic resolution by vicinities algorithm that has its origins in artificial intelligence, but which is used with much more success in the investigation of operations.

The tabu search, unlike other algorithms based on random techniques of neighboring solution searches, is characterised by its strategy based on the use of memory structures to escape local optimality, in which it can be trapped when moving from one solution to another in the solution space.

As such, this algorithm benefits from a 'memory' which stores the last moves made and which can be used to 'remember' solutions already explored. This 'memory' serves to impede the pathway to those solutions. The properties of this algorithm make it ideal to optimise the navigation of webpages, reducing waiting times and 'noise' (irrelevant information) in the search for information, and as such, there is a reduction in abandonment and consequently a better quality of navigation.

\section{Description of the Functioning of the Tabu Algorithm}

The hypothetical Internet user utilized for the study at a given moment considers the possibility of obtaining a mortgage loan for which he dedicates time to the navigation of the webpages of different online banks that he knows. The first accesses are to obtain information and compare it. Later he focuses on other aspects more related to contracting the product.

In the problem of navigation by a user of an online banking website, the penalty costs for delay and updating have been considered, both being of a linear type. The following webpage model will be used for the study (Figure 1).

Consider the maximum time dedicated to the navigation by the user of the site as 100 seconds (the average time spent by Internet users of financial and investment pages according to a study carried out between the months of April and June 2006 by Nielsen// Net Ratings, a company specialised in the measuring and analysis of Internet audience). This time will be divided into 5 possible areas: 1 (obtaining of information about products ) the maximum time dedicated to the navigation (n.a.t (navigation average time)) area 1 is 50 seconds, 2 (usage of simulators) n.a.t 40 seconds, 3 (entering of data) n.a.t 70 seconds, 4 (solving problems) n.a.t 80 seconds and 5 (contracting product) n.a.t 60 seconds. At time zero, $N$ accesses arrive at the page. Each access $i(1,2, \ldots . N)$ requires ti units of navigation time and has a delay penalty for each unit of time of $P_{i}$ starting from time zero; $S_{i j}$ is the updating cost of the page j immediately after access i. Two false accesses 0 and $N+1$ are included in each

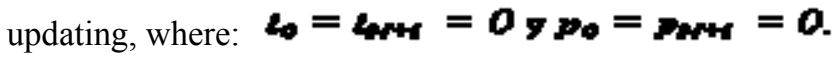

The costs $\boldsymbol{\varepsilon} \mathbf{y} \boldsymbol{\Psi} \boldsymbol{w} \boldsymbol{H}$ are considered as the costs of initial access and information refinement respectively. The navigation route for the page has the form: $\sigma=(0, \sigma(1), \sigma(2), \sigma(3), \ldots, \sigma(N), N+1)$, where $\sigma(i)$ is the access index in position $i$ of the page. The objective is to minimise the total of the delay and updating costs $\mathrm{D}(\sigma)$ for all the accesses $\mathrm{S}(\sigma)$. In mathematical terms, it is desired to minimise $F(\sigma)=D(\sigma)+S(\sigma)$, where:

$$
\begin{aligned}
& D(\sigma)= \sum_{i=1}^{N} d \sigma(i) p \sigma(i) \quad d \sigma(i)=\sum_{j}^{i-1} t \sigma(j), i=2, \ldots \ldots, N \cdots y \cdots d \sigma(1)=0 \\
& S(\sigma)=s 0, \sigma(1)+\sum_{i=1}^{N-1} s \sigma(i), \sigma(i+1)+s(\sigma(N), N+1
\end{aligned}
$$

With the objective of presenting the basic function of the Tabu Search, the problem of searching for an optimum navigation itinerary is considered. The initial solution is in agreement with the following order: $i<j$ implies that:

\section{$(4)<(p)$.}

For example, for a customer that gained access to information about a mortgage, the average time employed above the maximum foreseen would be (Table 1$)$.

For $\sigma=(0,3,1,2,5,4,6)$, accesses 0 and 6 are only false accesses and from here onwards are omitted. The tabu restrictions in order to prevent reverse moves would be formulated in the following form (Table 2). 
The tabu restrictions are not inviolable under all circumstances. When a tabu move provides a solution better than any other previously found, its tabu classification can be eliminated. The condition that permits this elimination is called the aspiration criterion. Finally a completion criterion can be produced on the basis of which a maximum number of iterations or a maximum number of steps that do not improve cost can be established. In this example, the criterion 2 in Table 1 is considered $(\sigma(i), i, \sigma(j), j)$ It impedes whatever move that results in an itinerary where whichever of the accesses $\sigma$ (i) occupies the position i or the work $\sigma$ (j) occupies the position $\mathrm{j}$. In addition, they have the following conditions (Table 3 ).

Next, the iterations with their order number indicated, the itineraries generated, the corresponding values of the objective function, the proposed value for the tabu move and the best admissible move to continue, will be presented in tables.

In this example all the neighbourhoods are examined and the best change is made when the minimum value in the objective function is obtained and it is not a tabu move or, if it is a tabu move, it must satisfy the aspiration criterion to be admissible. For this case, the aspiration criterion complies if the value of the objective function is better than all the previously found values.

The frequency matrix permits the 'history' of the procedure to be registered. It is utilised for the formation of the long term memory function and permits the diversification of the search as well as the possibility of directing the search 'closer' or 'further' from the regions explored.

Supposing that in a navigation session at least two admissible moves that give the best objective function values are registered, and either can be chosen, then the first is chosen and the itinerary selected acts as the start point for the following iteration. In the case where in a step itineraries are obtained with tabu moves but which satisfy the aspiration criterion, the start point for the following itinerary would be the itinerary with the smallest value in the neighborhood.

In general, if in any iteration there were no admissible points and the stop criterion had not been satisfied then an intensification and a diversification memory would have to be used in order to continue the search.

Normally the search is restarted from the best present solution. Some options would then be either to keep fixed the components or attributes that appear best or to change the neighbourhood.

\section{Conclusions}

In Internet navigation one of the principal goals is to achieve the most efficient information search, and this implies the reduction of search times and the elimination of unproductive routines or unhelpful or insufficient information for the Internet user. The adaptive memory of the tabu algorithm exploits the history of the problem resolution process by referring to four principal factors of: being recent, frequent and of high quality and influence. All these properties make it a method especially useful in the most efficient search for information.

The proposed algorithm Tabu Search provides a powerful tool to solve the job shop problem with the abandonment of web page navigation criterion. It offers very good accuracy, in comparison to other best known approaches, obtainable in a short running time on a modern PC. The great inconvenience in the use of heuristic methods is that it is not possible to know beforehand the quality of the solution they produce, thus making unknown the proximity of said solution to the global optimum.

\section{References}

Costa D. (1994). A Tabu Search Algorithm for Computing an Operational Time Table. European Journal of Operational Research, 76.

De Los Cobos Silva, S.G. (1994). La técnica de búsqueda tabú y sus aplicaciones. Tesis de Doctorado, Posgrado de Ingeniería, UNAM México.

Glover \& Laguna. (1997). Tabu Search. Kluwer Academic Publishers.

Glover, F. (1989). Tabu search - Part I, ORSA. Journal on Computing, 1, 190-206.

Glover, F. (1990). Tabu search - Part II, ORSA. Journal on Computing, 2.

Glover, Taillard, \& Laguna, De Werra (eds.). (1993). Tabu Search. Annals of Operations Research, 41.

Laguna, M, Barnes, J.W. \& Glover, F. (1990). Tabu Search for a Single Machine Schedulling Problem. Technical report, 7. Advanced Knowledge Systems Group of US, West Advanced Technologies.

Laguna, M, Kelly, J.P., González-Velarde, J.L., \& Glover, F. (1995). Tabu Search for the Multilevel Generalizad Assignment Problem. European Journal of Operational Research, 82. 
Laguna, M. (1993). A Guide to Implementing Tabu Search. Technical report, 10. Graduate School of Business and Administration. University of Colorado.

Semet F., \& Taillard E. (1993). Solving real-life vehicle routing problems efficiently using tabu search. Annals of Operations Research, 41.

Note

Note 1. A study carried out between the months of April and June of the 2006

Table 1. A customer data time navigation

\begin{tabular}{|c|c|c|c|c|c|c|}
\hline & $\begin{array}{c}1 \\
\text { Obtaining of } \\
\text { information } \\
\text { about the } \\
\text { products }\end{array}$ & $\begin{array}{c}2 \\
\text { Usage of } \\
\text { simulators }\end{array}$ & $\begin{array}{c}3 \\
\begin{array}{c}\text { Entering of } \\
\text { data }\end{array}\end{array}$ & $\begin{array}{c}4 \\
\text { Solving } \\
\text { problems }\end{array}$ & $\begin{array}{c}5 \\
\text { Contacting } \\
\text { product }\end{array}$ & \\
\hline A & $(14,19,24)$ & $(10,14,18)$ & $(12,16,20)$ & $(26,30,34)$ & $(19,23,27)$ & 100 \\
\hline & 50 & 40 & 70 & 80 & 60 & \\
\hline
\end{tabular}

Table 2. Tabu restrictions and attributes for interchange moves

\begin{tabular}{|l|l|l|}
\hline 1.- & $(\sigma(\mathrm{i}), \sigma(\mathrm{j}), \mathrm{i}, \mathrm{j})$ & $\begin{array}{l}\text { It prevents any move resulting from a permutation where } \sigma(\mathrm{j}) \text { has the position } \mathrm{i} \\
\text { and } \sigma(\mathrm{j}) \text { has the position } \mathrm{j}\end{array}$ \\
\hline 2.- & $(\sigma(\mathrm{i}), \mathrm{i}, \sigma(\mathrm{j}), \mathrm{j})$ & $\begin{array}{l}\text { It prevents any move resulting from a permutation where } \sigma(\mathrm{i}) \text { has the position } \mathrm{i} \\
\text { or } \sigma(\mathrm{j}) \text { has the position } \mathrm{jj}\end{array}$ \\
\hline 3.- & $(\sigma(\mathrm{i}))$ & It prevents $\sigma(\mathrm{i})$ from returning to position $\mathrm{i}$ \\
\hline 4.- & $(\sigma(\mathrm{i}), \mathrm{j})$ & Its prevents $\sigma(\mathrm{i})$ from returning to position $\mathrm{k}$ with $\mathrm{k}<=\mathrm{i}$ \\
\hline 5.- & $\sigma(\mathrm{i})$ & Its prevents $\sigma(\mathrm{i})$ from moving \\
\hline 6.- & $(\sigma(\mathrm{i}), \sigma(\mathrm{j}))$ & Its prevents $\sigma(\mathrm{i})$ and $\sigma(\mathrm{j})$ from moving \\
\hline
\end{tabular}

Table 3. Penalty and updating costs

\begin{tabular}{|c|c|c|c|c|}
\hline Areas & Duration & Penalization & Ratio & Natural order \\
$i$ & $t_{i}$ & $p_{i}$ & Durat/Penaliz & \\
\hline 1 & 19 & 7 & 2.71428 & $\sigma(4)$ \\
\hline 2 & 14 & 6 & 2.33333 & $\sigma(5)$ \\
\hline 3 & 16 & 5 & 3.2 & $\sigma(3)$ \\
\hline 4 & 30 & 3 & 10 & $\sigma(2)$ \\
\hline 5 & 23 & 2 & 11.5 & $\sigma(1)$ \\
\hline
\end{tabular}




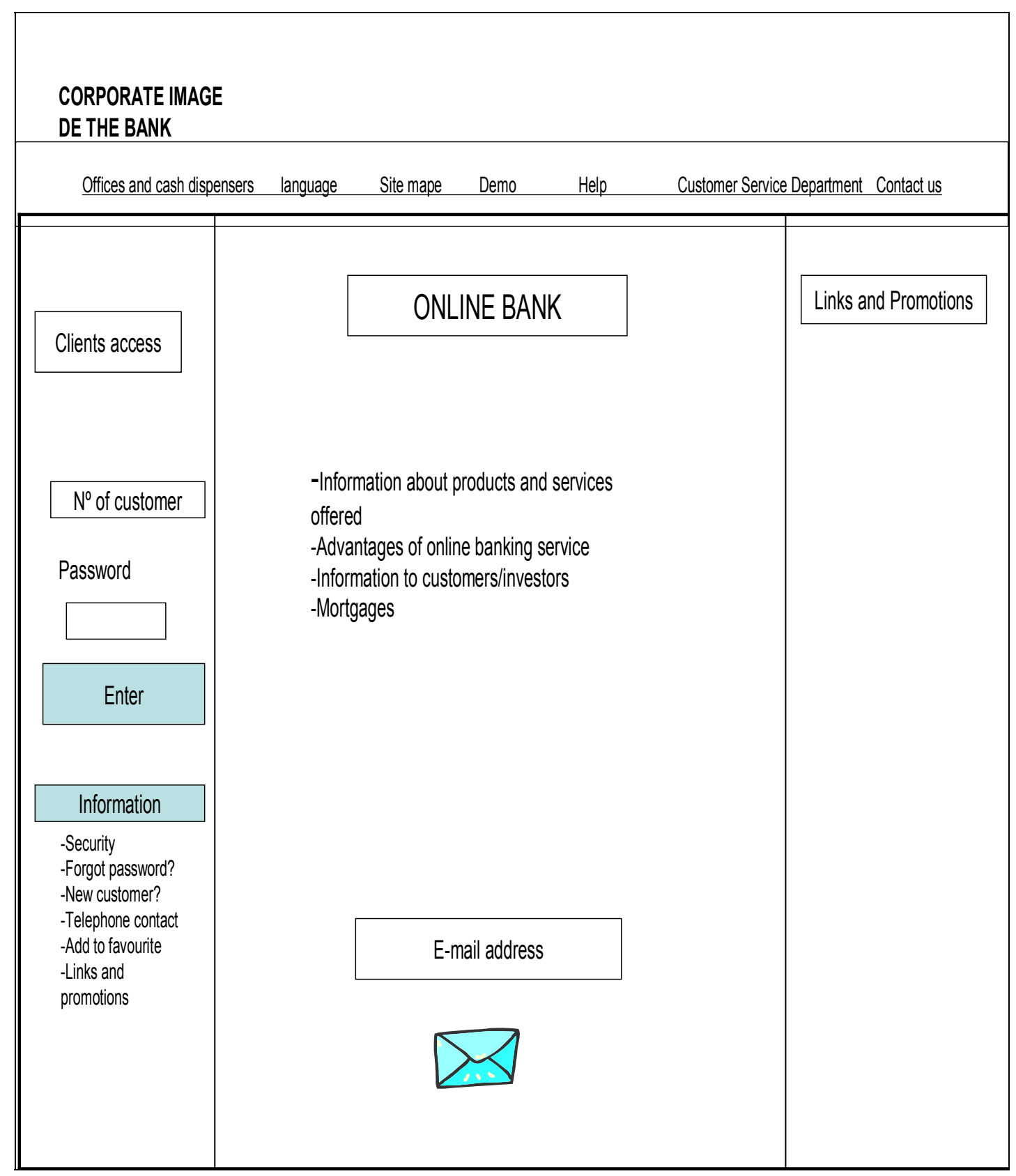

Figure 1. Web page model 